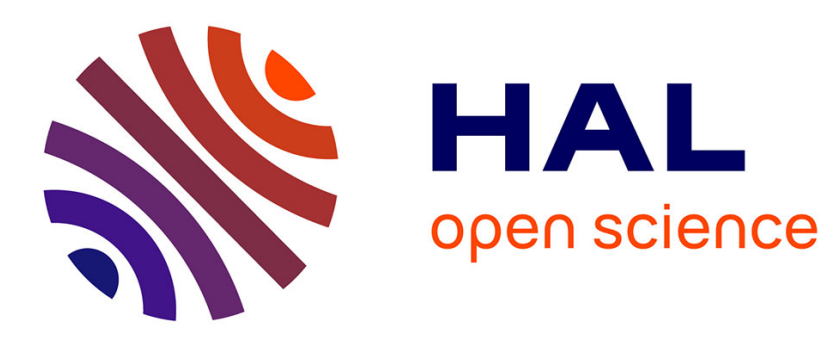

\title{
Numerical simulation of multipolar configuration and prepulse technique to obtain spatially reverse recruitment order
}

\author{
Mélissa Dali, Olivier Rossel, David Guiraud
}

\section{To cite this version:}

Mélissa Dali, Olivier Rossel, David Guiraud. Numerical simulation of multipolar configuration and prepulse technique to obtain spatially reverse recruitment order. EMBC: Engineering in Medicine and Biology Conference, Aug 2016, Orlando, United States. pp.5461-5464, 10.1109/EMBC.2016.7591962 . lirmm-01387130

\section{HAL Id: lirmm-01387130 \\ https://hal-lirmm.ccsd.cnrs.fr/lirmm-01387130}

Submitted on 25 Oct 2016

HAL is a multi-disciplinary open access archive for the deposit and dissemination of scientific research documents, whether they are published or not. The documents may come from teaching and research institutions in France or abroad, or from public or private research centers.
L'archive ouverte pluridisciplinaire HAL, est destinée au dépôt et à la diffusion de documents scientifiques de niveau recherche, publiés ou non, émanant des établissements d'enseignement et de recherche français ou étrangers, des laboratoires publics ou privés. 


\title{
Numerical simulation of multipolar configuration and prepulse technique to obtain spatially reverse recruitment order
}

\author{
Mélissa Dali ${ }^{1}$, Olivier Rossel ${ }^{1}$, David Guiraud ${ }^{1}$, IEEE EMBS Member
}

\begin{abstract}
In the context of functional electrical stimulation of peripheral nerves, the control of a specific motor or sensory functions may need selective stimulation to target the desired effect without others. In implanted stimulation, spatial selectivity is obtained using multipolar CUFF electrodes with specific spread of the current over each contact. Furthermore, electrical stimulation recruits large fibers before small ones, whereas the targeted function could be elicited by a specific fiber type i.e. fiber diameter.

In our work, numerical simulations were used to investigate the combination of multipolar configuration and prepulses, in order to obtain spatially reverse recruitment order. Multipolar stimulation provides efficient spatial selectivity, whereas subthreshold prepulses were used to reverse recruitment order with a reasonable increase of the injected charges. We compared several selective configurations combined with prepulses to show that some are able to guarantee both the spatial selectivity while one fiber's diameter can be preferentially activated.
\end{abstract}

\section{INTRODUCTION}

Functional electrical stimulation (FES) is a technique used to restore sensory or motor functions in case of neurological disorders for instance due to spinal cord injury, trauma or stroke. A nerve has multiple targets: to independently activate muscles, a control of activation by spatial selectivity of axons is required. Sunderland [15] published data about fascicular organization in peripheral nerves in distal regions, other investigations exist in the upper limb (ulnar nerve) [12], and lower limb (femoral nerve) [10]. In implanted stimulation, multicontact electrode such as CUFF electrodes are commonly used to activate the desired population of axons within the nerve. Numerical studies and experimentation have been carried out with CUFF electrodes to find configurations which best achieve spatial selectivity. Authors presented different configurations such as longitudinal tripolar [19], [14], field steering [16] [19] transverse tripolar [5], [14] among which Transverse Tripolar (TT) seemed to be the most selective one [9].

Another important issue in peripheral nervous system stimulation is fiber diameter selectivity. Unlike Natural Recruitment Order (NRO), conventional stimulation with a rectangular current pulse preferentially recruits large motor fibers over small ones. This phenomenon termed Inverse Recruitment Order (IRO) may cause undesirable effects such as muscle fatigue: rapid fatigable motor units innervated by large fibers are recruited before slow fatigue resistant motor units innervated by small fibers. Autonomic functions can

\footnotetext{
${ }^{1}$ French National Institute for computer science and applied mathematics (INRIA), University of Montpellier, CAMIN team, Montpellier, FRANCE. surname.namedinria.fr
}

also be specifically addressed by axons of a given diameter that is not the larger ones and thus may recquire also for this type of selectivity. Several approaches exist to achieve this goal: anodal block [2], high frequency block, slow rising ramp pulse [11] and subthreshold prepulses [7]. Subthreshold prepulses change the excitability of nerve fibers by increasing the activation thresholds of large fibers more than smaller ones. This technique has been validated in vivo on sciatic nerve of cat [7], and rat [18]. Simulation of subthreshold prepulses were performed with point source cathode [7], [6] and ring longitudinal tripole (LTR) configuration [6]. To our knowledge, in the context of subthreshold prepulses stimulation no studies investigate other multipolar configurations such as TT with a deeper analysis on the spatial selectivity. In this context, the objective of the present study is to obtain the recruitment of fibers with a specific diameter in a specific target area by combining simulations of both multipolar stimulation and prepulse approaches.

\section{Methods}

A 2-part process is used to determine nerve activation. First, we computed the potential field distribution produced by electrode through the nerve, then we applied this potential to a mammalian model of myelinated fibers to determine if activation occurs.

\section{A. Volume conductor model}

The potential field distribution generated by cuff electrode was obtained by solving Poisson's equation within the 3D model of the nerve under quasi-static conditions. The 3D Finite Element Model (FEM) of a cylindrical nerve and cuff electrode was implemented on COMSOL Multiphysics (COMSOL Inc. Burlington, MA). The nerve model was composed of a single fascicle with a $2.4 \mathrm{~mm}$ diameter, a perineurium and an epineurium with a $2.8 \mathrm{~mm}$ diameter surrounded by a layer of saline solution with $3 \mathrm{~mm}$ diameter. The perineurium thickness was set to $3 \%$ of the fascicle diameter according to [8]. Tissue electrical properties were based on [4]. Endoneurium was assumed to be anisotropic : conductivity were $0.06 \mathrm{~S} / \mathrm{m}$ in longitudinal direction $(\mathrm{Z})$ and $0.083 \mathrm{~S} / \mathrm{m}$ in transverse direction (XY), the conductivity of perineurium was $0.0015 \mathrm{~S} / \mathrm{m}$, that of epineurium was 0.083 $\mathrm{S} / \mathrm{m}$ and that of saline was $2 \mathrm{~S} / \mathrm{m}$. A cylindrical $(3 \mathrm{~mm}$ diameter) CUFF electrode with 12-pole (3 rows with 4 contacts) was modeled around the nerve. Adjacent ring separation was set to $1.5 \mathrm{~mm}$, contacts on a ring are placed at $0^{\circ}, 90^{\circ}$, $180^{\circ}$ and $270^{\circ}$ (Fig. 1). Poisson's equation was solved using COMSOL, the resulting potential was transferred to Matlab 
and interpolated along 333 fibers sampled on a grid (Fig. 1). Spacing in the radial direction $(\mathrm{X}, \mathrm{Y})$ was set to $0.1 \mathrm{~mm}$. Spacing on longitudinal direction $(\mathrm{Z})$ depends on internodal distance thus on fiber diameter.

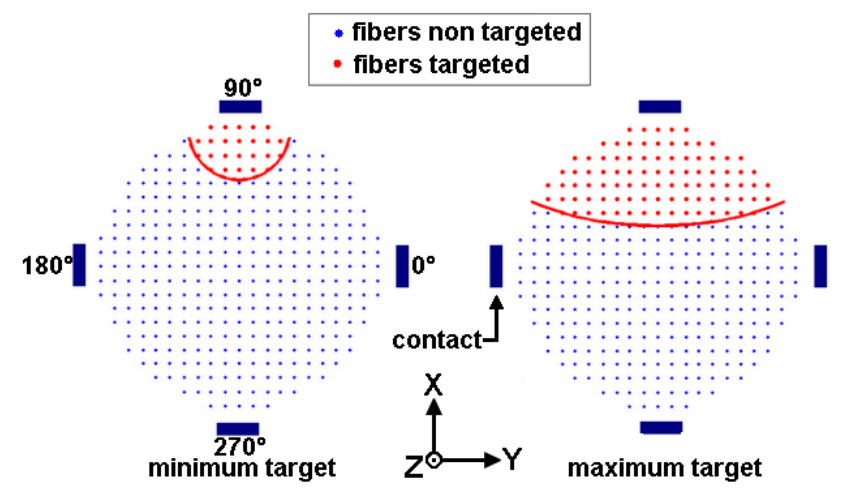

Fig. 1. Cross section of nerve model with 333 fibers. Electrode contacts are represented by their position around the nerve $\left(0^{\circ}\right.$ to $\left.270^{\circ}\right)$. Minimal and maximal target areas are represented

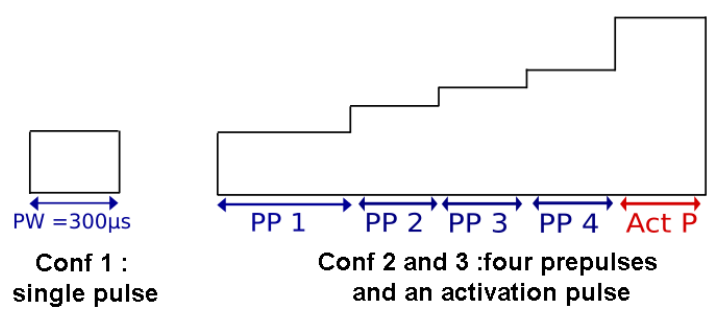

Fig. 2. Pulse waveform. Conf 1: single pulse, Conf 2 and 3: 4 prepulses (PP1 to PP4) followed by an activating pulse (ACT P)

\section{B. Nerve fiber model}

To represent motor axon response to stimulation, we implemented a cable model of axon based on [13]. Instead of Frankenhauser equations (amphibian model) we used CRRSS ones [3] (mammalian model) to describe non linear membrane dynamics. CRRSS equations were adapted to $37^{\circ} \mathrm{C}$ [17]. In the model of axon, the myelin is considered as a perfect insulator. Four axons with different diameters were simulated: $5 \mu \mathrm{m}, 10 \mu \mathrm{m}, 15 \mu \mathrm{m}$ and $20 \mu \mathrm{m}$. The central node of fibers was set under the central ring. Thresholds were then determined for all fibers inside the nerve.

\section{Current settings}

To get both spatial selectivity and a preferential activation of, in our example, $10 \mu \mathrm{m}$ fibers, we optimized current spreading between contact over time and pulse waveform with prepulses. First step was to determine an efficient current distribution on the contacts to obtain spatial selectivity. Second step was to set duration of prepulses. A long depolarizing prepulse increased the activation threshold of large fibers close to the cathode. Third step was to set number of prepulses. Our simulations showed that increasing number of prepulses expanded the area of inactivation and push back activation of large fiber further in the nerve. Duration and number of prepulses were optimized for the targets used in this study (data not shown). We used current configurations composed by 4 stepped prepulses (PP1 to PP4) followed by a depolarizing pulse (Act $\mathrm{P}$ ) and a single pulse in TT configuration was used as current configuration control. (Fig. 2 ). With this last configuration, only spatial selectivity is achieved in IRO.

1) multipolar configuration: First configuration ("Conf 1 ", current configuration control) was a single pulse of $300 \mu \mathrm{s}$ duration with TT configuration i.e. one cathode $90^{\circ}$, and 2 anodes at $0^{\circ}$ and $180^{\circ}$ (Fig. 1). Second configuration ("Conf 2") was composed of 4 prepulses with TT configuration (PP1 to PP4, Fig. 2) and one activating pulse (ACT P, Fig. 2) with TT configuration. Third configuration ("Conf 3") was composed of 4 prepulses (PP1 to PP4, Fig. 2) with LTR configuration, i.e., 4 cathodes in $0^{\circ}, 90^{\circ} 180^{\circ} 270^{\circ}$ with equal spreading of the current, and anodes on the external rings (i.e. all external contacts were short-circuited). The prepulses were followed by an activating pulse (ACT P, Fig. 2) with TT configuration.

2) Prepulse and pulse configuration: We increased number of prepulses, four instead of two compared to Grill [7] and Deurloo [6] to further elevate thresholds of large fibers close to the cathode (i.e. inactivate them) while activating fibers with smaller diameters. Increasing more than 4 prepulses was not needed according to our simulations. For "Conf 2" and "Conf 3", first prepulse duration was set to $500 \mu \mathrm{s}$ [7]. We however reduced duration of following prepulses to $200 \mu \mathrm{s}$ to limit the injection of charge. The duration of activating pulse was $200 \mu s$. To elicit medium fibers of $10 \mu \mathrm{m}$, we set prepulse parameters to reduce activation of $15 \mu \mathrm{m}$ and $20 \mu \mathrm{m}$ fibers diameter. First prepulse was set to be at $95 \%$ of the threshold of the first fiber of $15 \mu \mathrm{m}$ diameter activated, the same method is used for prepulse 2, 3 and 4. Activation pulse was observed for value between $-1 \mathrm{~mA}$ and $-3.5 m A$ on the cathode. For "Conf 1" (Fig. 2), duration of the pulse is set to $300 \mu \mathrm{s}$ and current varied from $-1 \mathrm{~mA}$ to $-1.7 m A$ on the cathode.

\section{Data analysis}

We focused on the maximum activation for fibers with $10 \mu m$ diameter. To quantify the effect of pulse shape and multipolar configuration on spatial and fiber diameter selectivity, we defined selectivity index for a target area on the cross section of the nerve. This target had an ellipsoidal form, centered on the top of the nerve (see Fig. 1). We varied the length of minor and major axis from 2 to $6 \mathrm{~mm}$ with a step of $0.1 \mathrm{~mm}$ for the major axis and 0.8 to $1.4 \mathrm{~mm}$ with a step of $0.05 \mathrm{~mm}$ for minor axis. For each diameter $D$, we defined 2 percentage of activation, in the targeted zone $n_{T A}(D)$, and in the non targeted zone $n_{N T A}(D)$. We defined a selectivity index as the difference between $n_{T A}(D)$ and $n_{N T A}(D)$ :

$$
S I(D)=n_{T A}(D)-n_{N T A}(D)
$$

The optimal current is determined as the amplitude of "Act P" giving the maximal SI of $10 \mu \mathrm{m}$ fibers diameter. Comparisons are given with this optimal current. 


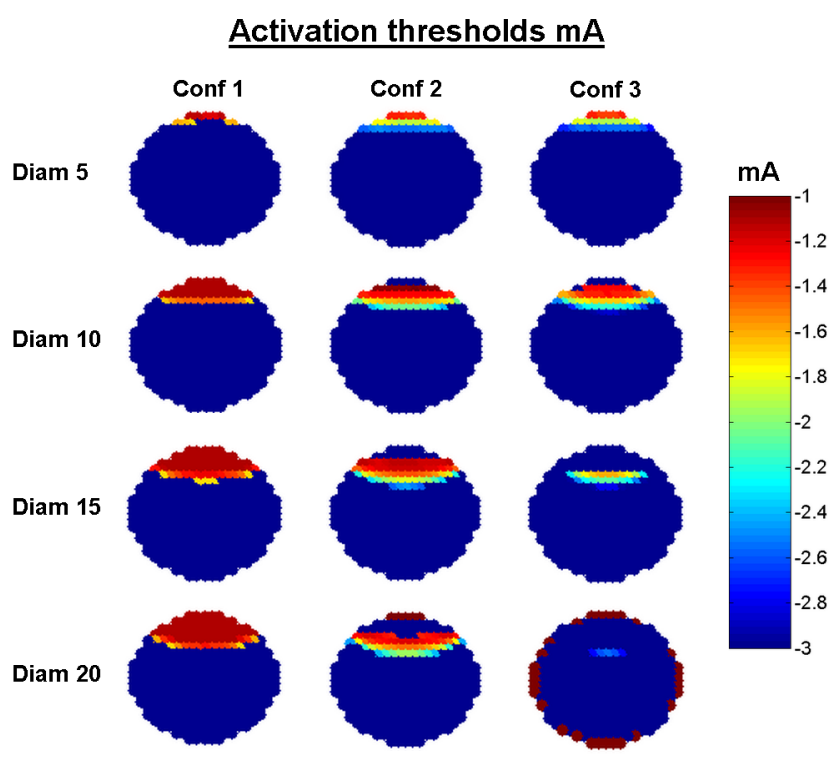

Fig. 3. Activation threshold for each configuration and each diameter. Conf 1: one pulse of TT configuration with $300 \mu s$ duration. Conf 2: 4 prepulses of TT configuration and a stimulating pulse of TT configuration. Conf 3: 4 prepulses of LTR configuration and one stimulating pulse of TT configuration. Color scale corresponds to thresholds of activation from -1 $m A$ and $-3 m A$ excepted for Conf $1-1 m A$ and $-1.7 m A$.

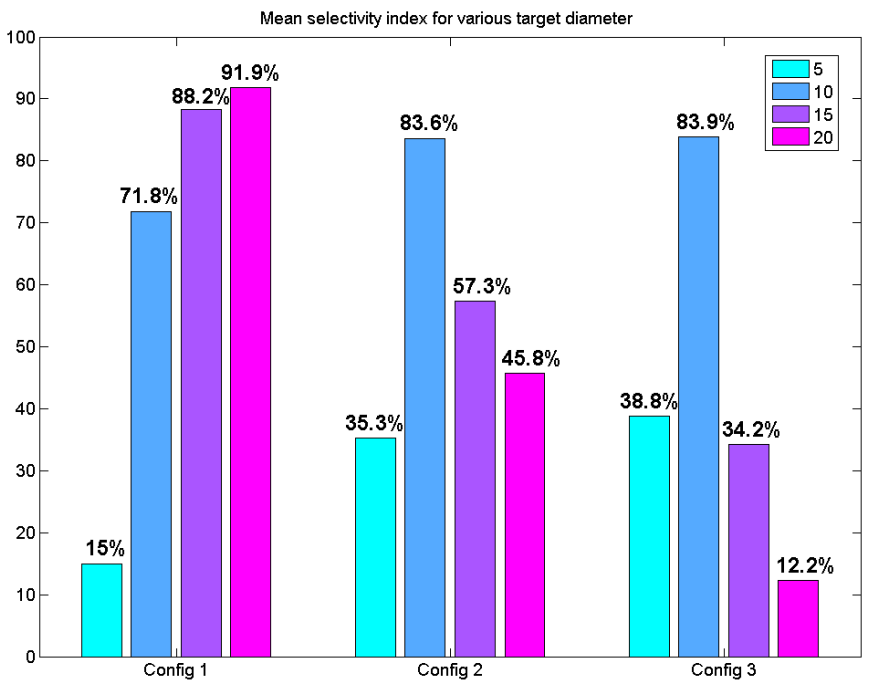

Fig. 4. Mean selectivity index for each configuration, and various target size. For each target, SI is optimal for fibers with $10 \mu \mathrm{m}$ diameter.

\section{RESULTS}

\section{A. Activation order}

The threshold of activation of each fibers for 4 different diameters and 3 configurations, is determined using dynamic nerve fiber model (Fig 3). For "Conf 2" and "Conf 3", we represented current needed for the activating pulse (ActP). Value of prepulses are listed on table I.

For Conf 1 , as expected, fibers with large diameter, i.e. $15 \mu \mathrm{m}$ and $20 \mu \mathrm{m}$, were preferentially recruited before fibers with smaller diameter i.e. $5 \mu \mathrm{m}$ and $10 \mu \mathrm{m}$.

Stepped TT prepulses ("Conf 2") increased thresholds, es-
TABLE I

CURRENT SETTINGS FOR PREPULSES

\begin{tabular}{|c||c||c|}
\hline Current pulse & TT & LTR \\
\hline PP 1 & $0.384 m A$ & $0.934 m A$ \\
\hline PP 2 & $0.533 m A$ & $1.335 m A$ \\
\hline PP 3 & $0.668 m A$ & $1.653 m A$ \\
\hline PP 4 & $0.852 m A$ & $2.017 m A$ \\
\hline
\end{tabular}

pecially for large fibers and reversed the current distance relationship close to the cathode as described in [7], [6]. However, activation of large fibers still occurs far from the cathode. In other words, small fibers were preferentially recruited and large fibers inactivated but this effect is limited to the proximity of the cathode with still activation of large fibers further. The "Conf 3" configuration is the most efficient to inactivate fibers of 15 and $20 \mu \mathrm{m}$ diameter over the whole nerve; even if a small amount of fibers of $20 \mu \mathrm{m}$ diameter were still activated at the extreme periphery of the nerve.

To sum up, "Conf 1" activated in IRO for current varying from $-1 m A$ to $-1.7 m A$, "Conf 2 " activated fibers in NRO but large fibers were activated further in the nerve for current varying from $-1 m A$ to $-3 m A$. "Conf 3 " activated fibers in NRO with minimal activation for the large fibers over the whole nerve, for current of the activating varying from $-1 m A$ to $-3 m A$ with a spatial selectivity for the $10 \mu m$ targeted fibers.

\section{B. Selectivity index}

Selectivity index (Eq 1) is computed for various targeted zones and range of current. We show the mean value of selectivity index of the optimal index (for $10 \mu \mathrm{m}$ fibers) found for each targeted zones. The bar chats in Fig. 4 represents this mean value for each configuration and each diameter. Fibers with 15 and $20 \mu m$ diameter were most often recruited with "Conf 1": respectively $88.2 \%$ (standard deviation, std $=9.8 \%$ ) and $91.9 \%(\mathrm{std}=5.3 \%)$ of activation, fibers with $10 \mu \mathrm{m}$ diameter were recruited with a mean of $71.8 \%(\mathrm{std}=17.0 \%)$. "Conf 2" and "Conf 3" had better selectivity index for fibers of diameter $10 \mu m: 83.6 \%(\mathrm{std}=6.5 \%)$ and $83.9 \%(\mathrm{std}=6.2 \%)$ respectively. The difference between "Conf 2" and "Conf 3" comes from selectivity index of large fibers: axons with 15 and $20 \mu \mathrm{m}$ diameter had higher selectivity index for "Conf 2": respectively $57.3 \%$ (std=6.6\%) and $45.8 \%(\mathrm{std}=10.0 \%)$ whereas selectivity index decreased with "Conf 3" : $34.2 \%$ $(\mathrm{std}=7.2 \%)$ and $12.2 \%(\mathrm{std}=5 \%)$ respectively. Selectivity index is not significant for axons with $5 \mu \mathrm{m}$ diameter for all configurations : $15.0 \%$ ("Conf 1"), 35.4\% ("Conf 2") 38.8\% ("Conf 3").

\section{DISCUSSION}

In this study, the combination of prepulse technique with multipolar configuration compared to a single pulse on TT configuration has been investigated by numerical study. As expected, the single TT pulse ("Conf 1") activates large fibers first under the cathode. Using TT prepulses ("Conf 2") activates large fibers further in the nerve with higher 
stimulation thresholds whereas it inactivates large fibers close to the cathode. With "Conf 3", it is possible to elicit activation of $10 \mu \mathrm{m}$ fibers diameter without activating large fibers of $15 \mu \mathrm{m}$ and $20 \mu \mathrm{m}$ diameter in a restricted target close to the cathode. "Conf 3" was designed to be the best configuration that elicited mainly $10 \mu \mathrm{m}$ fibers, even if some activation of large diameter fibers in the periphery occurs. On the one hand, the effect of external anodes was considered [6]: Block is possible with small contact spacing, when hyperpolarisation occurs close to the central node (node closest to cathode). Therefore, in our model, to block large fibers, longitudinal contact spacing was set to $1.5 \mathrm{~mm}$, same size of internodal distance of $15 \mu \mathrm{m}$ fibers. On the other hand, the 4 first prepulses increase the level of activation of all the fibers of $20 \mu \mathrm{m}$ within the nerve contrary to "Conf 2 " that limits this increase to axons close to the cathode. It could be explained by small axial gradient inherent to TLR configuration. Finally, last stimulation pulse with TT configuration, can be thus selective both as regard fiber diameter and location.

For all configurations, activation of $5 \mu \mathrm{m}$ fibers was not very significant (less 38.8\%) and cannot be managed separately, but as they have higher thresholds they are less activated in all configurations. Prepulses duration were set to increase thresholds of fibers with large diameter. We used a duration of $500 \mu \mathrm{s}$ for the first prepulse (PP 1) according to the study of Grill [7]. We found that is was enough to set following prepulses to $200 \mu s$ (PP2, PP3 and PP4) to elevate thresholds. Contrary to previous works, this leads to less injected charges with the same effect. Indeed simulations (not presented here) show that longer prepulses except the first one, is not needed.

The neuron model used was the same as [7], confirmed by experimental work. In our study, we assumed that nodes of Ranvier were aligned with the contacts. Simulations of Deurloo et al. [6] on longitudinal tripolar configuration shown that the effect of subthreshold persisted with any nodal position compared to the cathode, resulting in an increase of thresholds.

\section{CONCLUSION}

The results of this numerical study showed that we could obtain spatial reverse recruitment order using of multipolar configuration combined with prepulses. We are currently investigating multifasicular nerve together with other configurations such as steering current [16]. However, this study showed the need of powerful programmable waveform multioplar stimulator such as the one described in [1] to complete experimental assessment. Owing to this technology, we aim at designing experimental setup to validate these preliminary simulation results.

\section{ACKNOWLEDGEMENTS}

This work was supported by a grant from BPIFrance, "Intense", \#6354

\section{REFERENCES}

[1] David Andreu, David Guiraud, and Guillaume Souquet. A distributed architecture for activating the peripheral nervous system. Journal of neural engineering, 6(2):026001, apr 2009.

[2] G S Brindley and M D Craggs. A technique for anodally blocking large nerve fibres through chronically implanted electrodes. Journal of neurology, neurosurgery, and psychiatry, 43(12):1083-1090, 1980.

[3] S Y Chiu, J M Ritchie, R B Rogart, and D Stagg. A quantitative description of membrane currents in rabbit myelinated nerve. The Journal of physiology, 292:149-66, jul 1979.

[4] A.Q. Choi, J.K. Cavanaugh, and D.M. Durand. Selectivity of multiplecontact nerve cuff electrodes: a simulation analysis. IEEE Transactions on Biomedical Engineering, 48(2):165-172, feb 2001.

[5] K. E. I. Deurloo, Jan Holsheimer, and H. B. K. Boom. Transverse tripolar stimulation of peripheral nerve: a modelling study of spatial selectivity. Medical \& Biological Engineering \& Computing, 36(1):6674 , jan 1998 .

[6] Kirsten E. I. Deurloo, Jan Holsheimer, and Piet Bergveld. The effect of subthreshold prepulses on the recruitment order in a nerve trunk analyzed in a simple and a realistic volume conductor model. Biological Cybernetics, 85(4):281-291, oct 2001.

[7] W.M. Grill and J.T. Mortimer. Inversion of the current-distance relationship by transient depolarization. IEEE Transactions on Biomedical Engineering, 44(1):1-9, 1997.

[8] Yanina Grinberg, M.A. Schiefer, D.J. Tyler, and K.J. Gustafson. Fascicular Perineurium Thickness, Size, and Position Affect Model Predictions of Neural Excitation. IEEE Transactions on Neural Systems and Rehabilitation Engineering, 16(6):572-581, dec 2008.

[9] Thomas Guiho, Olivier Rossel, Guillaume Souquet, Hern??ndez Alfredo, Laure Laporte, Christine Azevedo-Coste, David Andreu, and David Guiraud. Toward complex multipolar selective neural stimulation. International IEEE/EMBS Conference on Neural Engineering, NER, 2015-July:569-572, 2015.

[10] Kenneth J Gustafson, Gilles C J Pinault, Jennifer J Neville, Ishaq Syed, John A. Davis Jr, Jesse Jean-Claude, and Ronald J Triolo. Fascicular anatomy of human femoral nerve: Implications for neural prostheses using nerve cuff electrodes. The Journal of Rehabilitation Research and Development, 46(7):973, 2009.

[11] K Hennings, L Arendt-Nielsen, S S Christensen, and O K Andersen. Selective activation of small-diameter motor fibres using exponentially rising waveforms: a theoretical study. Medical \& biological engineering \& computing, 43(4):493-500, 2005.

[12] M E Jabaley, W H Wallace, and F R Heckler. Internal topography of major nerves of the forearm and hand: a current view. The Journal of hand surgery, 5(1):1-18, jan 1980.

[13] Donald R McNeal. Analysis of a Model for Excitation of Myelinated Nerve. IEEE Transactions on Biomedical Engineering, BME23(4):329-337, jul 1976.

[14] Thomas N Nielsen, G a Mathijs Kurstjens, and Johannes J Struijk. Transverse versus longitudinal tripolar configuration for selective stimulation with multipolar cuff electrodes. IEEE transactions on bio-medical engineering, 58(4):913-9, apr 2011.

[15] Sydney Sunderland and Francis Walshe. Nerves and nerve injuries. 1968.

[16] J.D. Sweeney, D.A. Ksienski, and J.T. Mortimer. A nerve cuff technique for selective excitation of peripheral nerve trunk regions. IEEE Transactions on Biomedical Engineering, 37(7):706-715, jul 1990.

[17] JD Sweeney, JT Mortimer, and D Durand. Modeling of mammalian myelinated nerve for functional neuromuscular stimulation. In IEEE 9th Annual Conference of the Engineering in Medicine and Biology Society, volume 3, pages 1577-1578, 1987.

[18] a.I I. Van Bolhuis, J. Holsheimer, and H.H.C.M H C M Savelberg. A nerve stimulation method to selectively recruit smaller motor-units in rat skeletal muscle. Journal of Neuroscience Methods, 107(1-2):87-92, 2001.

[19] C Veraart, W M Grill, and J T Mortimer. Selective control of muscle activation with a multipolar nerve cuff electrode. IEEE transactions on bio-medical engineering, 40(7):640-53, jul 1993. 